\title{
TV/Series
}

9 | 2016

Guerres en séries (I)

\section{La septième saison de 24 heures chrono : illusion d'un tournant moral et éthique?}

\section{Alexis Pichard}

\section{OpenEdition}

1 Journals

Édition électronique

URL : http://journals.openedition.org/tvseries/1268

DOI : $10.4000 /$ tvseries. 1268

ISSN : 2266-0909

Éditeur

GRIC - Groupe de recherche Identités et Cultures

Référence électronique

Alexis Pichard, "La septième saison de 24 heures chrono : illusion d'un tournant moral et éthique ?», TV/Series [En ligne], 9 | 2016, mis en ligne le 01 juin 2016, consulté le 10 décembre 2020. URL : http:// journals.openedition.org/tvseries/1268; DOI : https://doi.org/10.4000/tvseries.1268

Ce document a été généré automatiquement le 10 décembre 2020.

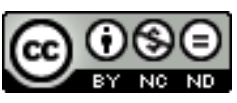

$T V /$ Series est mis à disposition selon les termes de la licence Creative Commons Attribution - Pas d'Utilisation Commerciale - Pas de Modification 4.0 International. 


\title{
La septième saison de 24 heures chrono : illusion d'un tournant moral et éthique?
}

\author{
Alexis Pichard
}

«Les règles sont ce qui nous rend meilleurs. - Pas aujourd'hui. "

24 heures chrono, 7.8 .

1 Pas un échange ne paraît mieux représenter la septième saison de la série 24 heures chrono que cette joute verbale entre l'agent vertueux du FBI Larry Moss (Jeffrey Nordling) et l'agent voyou Jack Bauer (Kiefer Sutherland). Pour l'un, les règles doivent être respectées en tout temps, quelle que soit la situation car elles garantissent la pérennité de notre humanité. Pour l'autre, dans certaines circonstances, les règles peuvent être contreproductives et rendre moins efficace. Dans 24 heures chrono, ces circonstances exceptionnelles ont trait au terrorisme national, moteur principal de l'intrigue, qui nécessiterait ainsi une suspension de certaines règles, notamment en termes de libertés individuelles, afin d'être contré. La série demande alors aux spectateurs si tous les moyens sont bons pour protéger la sécurité intérieure et les invite à se projeter dans la diégèse.

2 Si l'implication morale du spectateur n'est pas une nouveauté à l'échelle de la série puisque nous sommes en position de juger chaque décision prise par les personnages, la mise en scène des options morales qui sous-tendent ces décisions est, elle, inédite. Pour ce faire, la septième saison organise un débat sur ses thèmes récurrents (le recours aux méthodes coercitives, la logique jusqu'au-boutiste en matière de contreterrorisme, le piétinement des libertés individuelles pour le bien commun etc.), se confrontant aux ambiguïtés idéologiques qui habitent la série et, par là même, aux critiques dont elle a pu être victime.

3 Traditionnellement cataloguée (à tort) comme réactionnaire ou conservatrice, 24 heures chrono a longtemps été perçue comme un monolithe univoque figé dans la présidence 
Bush. En cause, une monstration fantasmée - car efficace - de la guerre contre le terrorisme et des liens étroits entre l'un des créateurs et le parti républicain. Aussi, bien que la série soit travaillée dès ses débuts par une ambivalence idéologique qui l'éloigne parfois de la politique gouvernementale, la saison 7 va venir nettement rompre avec ces conceptions en se mettant à jour avec le monde réel. Alors qu'Obama vient d'être élu et que celui-ci dresse un bilan de son prédécesseur, 24 heures chrono en fait de même en organisant littéralement son propre procès. Cette intrusion du réel, des débats moraux contemporains, du zeitgeist des années Obama justifient ainsi que l'on considère la septième saison comme un virage important dans l'histoire de la série.

Ainsi, après en avoir étudié le contexte de production et montré que 24 heures chrono est bien une œuvre évolutive, déterminée par les changements sociétaux, nous verrons comment la saison 7 s'adapte à une Amérique post-Bush et si les réponses qu'elle apporte en termes de morale évoluent. Afin d'en rendre compte, nous nous attarderons sur le traitement de la torture et sur la tension déontologisme/utilitarisme en nous demandant si Jack peut enfin mettre à mal le terrorisme tout en respectant les lois.

\section{La nécessité d'un renouveau : étude du « contexte génétique » de la saison 7}

5 À l'instar de Jerrold Levinson, pour qui l'œuvre d'art ne peut être pleinement comprise que si elle est appréhendée à l'aune de son contexte de production (historique, politique, artistique etc.), comprendre les évolutions qui surviennent lors de la septième saison de 24 heures chrono ne peut se faire qu'à l'issue d'un replacement de la série dans l'Histoire. Levinson rappelle, en effet, que :

Les œuvres d'art sont essentiellement des objets incorporés dans l'histoire et ils n'ont jamais un statut d'art, ni de propriétés esthétiques manifestes, ni de significations artistiques définies, ni d'identité ontologique déterminée, en dehors ou indépendamment d'un contexte génétique qui en fait les œuvres d'art qu'elles sont ${ }^{1}$.

6 Par l'étude du «contexte génétique » des œuvres d'art, le philosophe américain entend s'attarder sur les conditions de leur genèse afin d'aiguiller notre interprétation critique. C'est ce travail préliminaire et essentiel que nous nous proposons de mener dans un premier temps en dégageant trois types de contextes pertinents : le contexte réceptif, le contexte historique et le contexte créatif, tous trois étant intimement liés.

7 La septième saison de 24 heures chrono apparut en janvier 2009 sur les écrans de la Fox, soit plus d'un an et demi après la sixième. Comme le relate le documentaire 24/7: the Untold Truth ${ }^{2}$, cette nouvelle journée infernale de la vie de Jack Bauer fut le résultat d'un long et laborieux parcours. Lors de l'épilogue de la sixième saison, Jack se retrouvait au bord d'un précipice, se tenant face à la mer et à l'horizon doré d'un nouveau jour. Le temps était venu pour lui de rendre les armes et de renoncer à son martyr d'Atlas moderne - sauver l'Amérique en vingt-quatre heures chrono - à l'issue d'une journée infernale que beaucoup jugèrent décevante.

8 Certes, cette sixième saison reçut un accueil médiatique plutôt favorable mais elle vit son audience s'éroder au fil de l'année pour accuser une baisse de $23 \%$ entre les scores du season premiere $^{3}$ et ceux du season finale ${ }^{4}$. Bien que 24 heures chrono fût assurée de ne pas disparaître des écrans, la Fox l'ayant renouvelée pour deux saisons supplémentaires, elle 
devait néanmoins opérer des changements conséquents pour partir à la reconquête de son public. L'image du précipice prenait alors une dimension métafictionnelle : Jack était loin d'être le seul à se demander ce qui l'attendait.

9 Les premiers scripts de la saison 7 prévoyaient de suivre la quête rédemptrice de Jack en Afrique en parallèle avec l'enquête de Renee Walker (Annie Wersching), agent du FBI, à Washington. Après trois épisodes, la saison exécutait un saut dans le temps de douze heures et rapatriait Jack à Washington où il rencontrait Renee. Le temps réel restait le principe fondamental de la série tout en autorisant, pour la première fois de son histoire, une ellipse conséquente. Finalement, cet incipit fut abandonné même si l'idée d'un bond dans le temps sera finalement développée dans la saison neuf diffusée en 2014. Deux autres postulats furent alors envisagés. Les scénaristes pensèrent à une intrigue montrant Jack sous couverture dans un rôle de méchant puis l'imaginèrent au cœur d'une commission sénatoriale visant à le mettre face aux actes qu'il a commis. C'est finalement cette dernière idée qui fut exploitée avec pour objectif métafictionnel de répondre aux attaques que la série subissait alors. Comme le souligne David Fury, scénariste et producteur de 24 heures chrono: "On pouvait ainsi répondre aux critiques qu'on nous avait adressées durant la saison six, disant que nous glorifiions la torture, ce qui n'a jamais été notre but mais certains l'ont perçu ainsi ${ }^{5}$."

10 Car, en plus des critiques sur l'usure de sa forme, 24 heures chrono se retrouvait également attaquée une nouvelle fois, mais d'une manière bien plus soutenue, sur son rapport complexe à la torture. Comme le rappelle Jean-Baptiste Jeangène Vilmer, « 24 a depuis ses débuts suscité de vives critiques mais, jusqu'à fin 2008, son équipe n’a que très rarement considéré la torture comme un problème moral digne d'être discuté au sein même de la série $^{6}$.» Si ce constat apparaît légèrement excessif, il est vrai que son recours est le plus souvent institutionnalisé comme le prouve la saison six où chaque interrogatoire donne lieu à une scène de torture. D'ailleurs, le terme « interrogatoire » devient synonyme de « torture » : celui qui interroge torture, nécessairement.

11 Les critiques qui s'élevaient alors à l'encontre de 24 heures chrono furent galvanisées par la parution d'une enquête sur la série parue en février 2007 - durant la saison 6 - dans le magazine libéral The New Yorker'. Au cours d'un portait exhaustif de Joel Surnow, créateur de la série, la journaliste Jane Mayer met au jour les liens étroits que celui-ci entretient avec l'administration Bush, Surnow étant un féroce républicain, et l'idéologie conservatrice qu'il fait planer sur les aventures de Jack Bauer. Mayer prend pour exemple le cas de la torture dont elle montre qu'elle est sciemment utilisée à des fins politiques dans la série. Par Surnow, du moins. Ce dernier avoue, dans une interview accordée à la journaliste, croire à la légitimité de la torture en des circonstances extrêmes : « On dit que la torture ne marche pas. Je n'en crois rien. Je ne pense pas qu'il soit honnête de dire que si un être qui vous est cher était retenu prisonnier, et que vous aviez cinq minutes pour le sauver, vous ne tortureriez pas. Dites-moi, que feriez-vous? Si quelqu'un détenait l'un de mes enfants, ou bien mon épouse, j'espère que je torturerais cette personne. Il n'y a rien, rien, que je me refuserais de faire ${ }^{8}$. " L'article du New Yorker prit une telle résonnance dans le débat médiatique que les scénaristes furent, en quelque sorte, contraints d'y répondre9. Alors que la septième saison commençait à prendre forme, malgré le retard accumulé du fait d'un scénario palimpseste, la production fut arrêtée en novembre 2007 par la grève des scénaristes qui contraignitt la Fox à un report d'un an de la diffusion de la série avec tous les risques encourus par un tel hiatus. Lorsque le tournage reprit en février 2008, Joel Surnow décida de quitter son poste de showrunner pour se lancer dans d'autres projets ${ }^{10}$. 
Ce départ ne fut pas sans conséquence. Comme nous venons de le mentionner, Surnow est un républicain convaincu, en grande partie responsable des critiques développées à l'encontre de 24 heures chrono au cours de la sixième saison. Même s'il resta rattaché à la série en tant que consultant, il fut remplacé en qualité de showrunner par Howard Gordon, démocrate modéré. Ce changement politique à la tête de la série, bien que les scénaristes fussent de toute obédience, peut ainsi expliquer la tournure plus libérale des dernières saisons.

En mars 2008, la Fox fit part de son inquiétude à l'équipe créative quant au long hiatus entre la sixième et septième saison, souhaitant diffuser un aperçu des nouveaux épisodes afin d'attiser la curiosité du public. En lieu et place, les scénaristes optèrent pour un téléfilm qui servirait de transition. Pour ce faire, ils reprirent les premiers jets qu'ils avaient rédigés et exploitèrent l'idée originelle de voir Jack en Afrique. Baptisé Redemption , le téléfilm fut tourné en Afrique du Sud pour être diffusé en novembre 2008. On découvre donc Jack au Sangala, pays d'Afrique fictif, s'affairant à la construction d'une école pour les enfants défavorisés avec le soutien de l'ONU. Jack se trouve alors à l'abri du monde avant qu'il ne reçoive une assignation à comparaitre devant une commission sénatoriale pour détention et torture de prévenus et qu'il soit contraint à revenir aux États-Unis. Les temps ont changé, celui qui a sauvé l'Amérique par tous les moyens doit à présent répondre de ses actes, laissant présager un retour de l'éthique et de la morale à mettre en relation avec l'arrivée au pouvoir de Barack Obama.

14 Ce dernier fut élu président des États-Unis le 4 novembre 2008 contre le candidat républicain John McCain et cristallisa alors tous les espoirs d'une nation éreintée par sept années de guerre contre le terrorisme, aussi bien sur le sol américain qu'au MoyenOrient, de scandales, et de mensonges d'État. Comme Jack, l'Amérique cherchait aussi la rédemption après, notamment, l'échec des guerres afghane et irakienne et les scandales de Guantanamo et d'Abou Ghraib. À l'ère de la paranoïa collective qu'incarnait Bush devait succéder l'apaisement porté par Obama. Durant la campagne présidentielle, celuici promit des changements de fond. Opposé à la logique utilitariste de Bush et au manque de transparence qui l'accompagna, il envisagea de mettre un terme à la torture, aux guerres injustifiées, au piétinement des libertés individuelles au nom de la sécurité nationale et, de manière hautement symbolique, annonça la fermeture de Guantanamo ${ }^{11}$. Cette volonté de se démarquer des années Bush fut réaffirmée dans son discours d'inauguration programmatique de janvier 2009 qui célèbre l'émergence d'une Amérique désireuse de paix et de d'exemplarité :

À tous les peuples et gouvernements qui nous regardent aujourd'hui, des plus grandes capitales jusqu'au petit village où mon père est né, sachez que l'Amérique est l'amie de chaque pays, de chaque homme, femme et enfant en quête d'un avenir de paix et de dignité et que nous sommes prêts à nouveau à endosser le rôle de dirigeant ${ }^{12}$.

C'est donc dans ce contexte que fut diffusé Redemption qui sert de prologue à la septième saison en posant les fondements encore timides d'une ère nouvelle. Coïncidence des dates, le téléfilm survint à peine deux semaines après l'élection d'Obama. On retrouve dans cet épisode "hors-série » toute l'atmosphère du début de mandat du quarantequatrième président des États-Unis, que ce soit par l'arrivée au pouvoir de la démocrate Allison Taylor (Cherry Jones), présentée comme une déontologue idéaliste, ou à travers le rapatriement de Jack à Washington afin qu'il soit jugé pour ses actes. À travers son procès, c'est bien celui des années Bush qui s'opère. 


\section{La torture en accusation : le débat moral à l'épreuve d'une nécessité narrative}

16 Les séries télévisées, par leur mode de production et de diffusion, ont toujours su capter l'air du temps. Dans l'après 11 Septembre, leur réactivité fut instantanée. En effet, il ne fallut que quelques semaines aux scénaristes pour inclure la tragédie que venait de vivre la nation américaine à leurs productions, contrairement au cinéma qui mit quelques années avant de s'emparer de l'événement ${ }^{13}$. Ainsi, le zeitgeist de la guerre contre la terreur lancée par le président Bush le 20 septembre 2001 imprègna un paysage télévisuel qui se fit alors premier soutien de la Maison-Blanche : les séries épousèrent l'idéologie de l'administration Bush et œuvrèrent à son acceptation par le public américain. Cette tendance, particulièrement remarquable dans le genre thriller, s'ébranla après 2005 alors que grandissait la contestation populaire vis-à-vis de la guerre en Irak ${ }^{14}$.

17 Parmi les moyens de prévention du terrorisme défendus par l'administration Bush et certains éditorialistes, la torture se trouva ainsi évoquée et pratiquée dans de nombreux dramas abordant le terrorisme, que ce soit Agence Matrix (Threat Matrix; ABC, 2003-2004), DOS: Division des opérations spéciales (E-Ring; CBS, 2005-2006) ou encore Sleeper Cell (Showtime, 2005-2006). Néanmoins, par les critiques vives qu'elle lui values, la torture resta surtout associée à 24 heures chrono. L'offre télévisuelle et le message politique délivrés dans l'après 11 Septembre créèrent donc un contexte favorable à l'acceptation populaire de la torture comme moyen légitime et fiable de prévenir la menace terroriste. Dans les programmes diffusés entre vingt-heures et vingt-deux heures, les actes de torture passèrent de moins de quatre par an avant le 11 Septembre à plus d'une centaine ${ }^{15}$ . 24 heures chrono se plaça à la tête de cette tendance, cumulant 67 scènes de torture au cours des cinq premières saisons, avec un paroxysme atteint lors de la quatrième, comportant en moyenne des actes de torture dans plus de la moitié des épisodes ${ }^{16}$.

Une telle omniprésence de la torture n'est pas sans conséquence. Premièrement, elle banalise l'utilisation de telles méthodes coercitives en nous habituant au corps meurtri certains ont d'ailleurs parlé de torture-porn pour définir l'accoutumance que l'on peut développer face à l'horreur de ces scènes ${ }^{17}$ - et en variant les supplices. Ensuite, elle démontre que ces méthodes sont d'une immuable efficacité, à de rares exceptions près. Enfin, elle implique que tous les moyens, même immoraux, sont bons en des circonstances extrêmes. Ce sont ces trois messages qui sont transmis au public par le truchement de 24 heures chrono et qui participent de la redéfinition de la notion de torture. Qu'elle soit immorale et illégale importe peu puisqu'elle est de toute manière nécessaire, donc incontournable, du fait de son efficacité. Or, le problème qui se pose est le suivant : dans notre monde réel, la torture est loin d'être un moyen fiable afin d'obtenir des informations ${ }^{18}$. Cette réalité n'atteignit pourtant pas le monde de Bauer ou, du moins, pas avant la septième saison.

Dans 24 heures chrono, la torture est exécutée par l'ensemble des personnages, qu'ils soient de gentils agents de la CTU ou de méchants terroristes. C'est en soi une nouveauté des productions de l'après 11 Septembre au sens où, par le passé, les tortionnaires étaient entièrement maléfiques. Par contraste, les héros sont maintenant appelés à commettre le même outrage moral pour le bien commun. Comment les distinguer encore des terroristes qu'ils combattent? 
Bien que la torture en elle-même puisse être considérée comme immorale, puisqu'elle implique une douleur infligée par un sujet conscient sur autrui et qu'elle considère ce dernier comme un moyen, ce qui bafoue le précepte kantien selon lequel autrui ne doit être envisagé seulement comme un moyen, son utilisation par les agents de la CTU, par les divers présidents et, surtout, par Jack lui confère pourtant un caractère moral dans une perspective conséquentialiste.

D'une part, la torture est toujours pratiquée par devoir moral à l'aune des conséquences bénéfiques qu'elle peut engendrer. Quand Jack torture Syed Ali dans la deuxième saison, c'est dans le seul et unique but de savoir où est la bombe nucléaire qui menace de ravager Los Angeles. Il réfute tout sadisme, seul son devoir moral d'agent gouvernemental et d'être humain motive son piétinement des conventions de Genève de 1949. Il l'affirme à Syed Ali : «Je vous hais pour ce que vous m'obligez à faire» (2.12). Cette conception utilitariste de la torture n'est pas sans rappeler la morale développée par le philosophe Jeremy Bentham relative à la torture, lequel en légitimait l'utilisation dans certains cas extrêmes ${ }^{19}$.

D'autre part, la torture est le plus souvent pratiquée par des individus moraux qui en usent mais n'en abusent pas. Jack est à nouveau un bon exemple puisqu'il n'utilise la torture qu'en dernier ressort, qu'il n'en tire aucun plaisir et, surtout, qu'il en connaît les conséquences. En effet, il est prêt à être jugé pour ses actes, il ne soustrait nullement à la loi, ce qu'il réaffirme au début de la septième saison. Ce qui le rend aussi moral, c'est aussi son sens du sacrifice. Pour faire ce qui est nécessaire, il est prêt à sacrifier son humanité. Il endosse alors le rôle de martyr, sauvant les autres âmes de la damnation en commettant tous les crimes, et son sacrifice dépasse le cadre de la moralité puisqu'il est aussi prêt à se sacrifier physiquement.

Comme l'explique Timothy Dunny: «Nous sommes enclins à penser que si Jack torture quelqu'un, même un innocent, c'est acceptable car il est lui-même prêt à souffrir et à être torturé si nécessaire ${ }^{20}$. P Parce qu'il connaît le mal et qu'il l'a enduré, Jack est capable de faire le meilleur choix entre les différents préceptes moraux qui s'offrent à lui. Les " gentils » torturent donc principalement par nécessité avec une perspective téléologique morale et altruiste : sauver l'Amérique. Cela n'empêche bien sûr pas des débordements, des ratés, comme le montrent les quelques scènes de torture d'innocents (Nadia Yasir dans la saison six, Jack dans la saison huit etc.) qui insistent sur les limites d'une telle politique.

istes, quant à eux, ont un recours à la torture plus direct et systématique. La phase de dialogue est expéditive et laisse rapidement la place à la menace et aux sévices. S'ils torturent également pour obtenir des informations, il leur arrive aussi de torturer par revanche ou par pur sadisme. C'est ce qui arrive à Jack lorsqu'il est remis aux mains du terroriste Abu Fayed (Adoni Maropis), dont le premier réflexe est de l'attacher à une chaise pour le torturer (6.1). Autre différence notable avec les " gentils », la torture, chez les terroristes, précède toujours la mort. On ne compte plus les exemples où un tortionnaire promet d'épargner la vie de son captif ou celle de sa famille s'il lui révèle les informations qu'il veut savoir avant de se dédire finalement. Si la torture est immorale selon les préceptes kantiens, les terroristes parviennent à lui apporter un surplus d'inhumanité en ne tenant pas leurs promesses initiales.

Comme nous l'avons vu plus haut, l'élection d'Obama conjuguée à la parution de l'article de Jane Mayer dans le New Yorker poussèrent les scénaristes de 24 heures chrono à apporter 
des inflexions idéologiques à leur traitement de la torture. Plus qu'une profonde remise en cause menant à l'abandon des méthodes coercitives, la saison 7 propose surtout un débat moral en replaçant la torture dans une Amérique non plus régie par le calcul utilitariste mais par le droit et les principes fondateurs de la nation. La délocalisation de l'intrigue de Los Angeles à Washington sert cette ambition. Capitale fédérale des ÉtatsUnis créée officiellement par la Constitution de 1787, elle représente à elle seule l'idéal démocratique dont la nation s'est voulue le symbole lors de sa fondation. Par essence, Washington est le lieu du droit et du politique en réunissant les sièges des trois « Freins et Contrepoids» (Checks and Balances) : la Cour Suprême (pouvoir judiciaire), Le Président (pouvoir exécutif) et le Congrès (pouvoir législatif).

Ainsi, 24 heures chrono semble d'emblée signifier un changement fort de perspective qui va se trouver exacerbé dès le début de la première heure où l'on retrouve Jack au tribunal face au Sénateur Mayer (Kurtwood Smith) ${ }^{21}$ (7.1). Si cette scène n'est pas une surprise pour le spectateur, puisqu'elle était déjà annoncée dans Redemption, elle se révèle néanmoins inédite car jamais auparavant Jack n'avait eu à rendre des comptes à la justice. Une commission sénatoriale a ainsi été convoquée afin d'étudier les cas de violation des droits de l'homme par la CTU dont nous apprenons le récent démantèlement. Très vite, le sénateur Mayer aiguille l'audience vers le sujet brûlant qui anime le débat, la torture, et confronte le premier témoin de la journée, Jack Bauer. Les temps ont changé : à la torture institutionnalisée des années Palmer (aussi bien David que Wayne), notamment, succède le règne de la loi et de la morale de la présidente fraîchement élue Allison Taylor. Durant sa campagne, celle-ci a soutenu l'interdiction de la torture de même qu'elle a œuvré à une réforme en profondeur des agences de renseignement, contribuant de fait à la disparition de la CTU (7.11). Taylor réaffirme les principes fondateurs des États-Unis et le rôle de guide moral qu'ils doivent tenir à l'échelle du monde. Pratiquer la torture serait une marque d'hypocrisie : si l'Amérique se veut modèle, elle doit agir comme telle.

De fait, en supprimant la CTU, Taylor entend purger l'Amérique de ses relents barbares. Le FBI, à qui incombe dorénavant la lourde tâche du contre-terrorisme, est présenté en parangon de déontologisme. Les locaux lugubres et souterrains de la CTU cèdent la place à des bureaux ouverts et lumineux, signe de transparence. Les agents utilitaristes et indisciplinés sont remplacés par des agents éthiques et moraux à l'image de Larry Moss, de Janis Gold (Janeane Garofalo) ou encore de Renee Walker. Enfin, et surtout, la torture est proscrite, devenue impératif catégorique au sens où toute suggestion de son usage conduit à une réponse définitive, parfois sans réelle argumentation. Ainsi, quand Tony Almeida (Carlos Bernard), désormais terroriste, refuse de collaborer avec le FBI, Renee suggère à Larry de recourir à des moyens plus musclés (7.3) :

RENEE. Non, je suggère qu'on emploie la manière forte.

LARRY. La torture donc

RENEE. Il y a des méthodes coercitives auxquelles nous n'avons pas pensé. Les drogues...

LARRY. J'ai du mal à croire que tu veuilles avoir cette conversation. (...) Ton idée est démente. C'est illégal et ça ne se fait plus.

Non seulement la torture se trouve renvoyée au domaine de la barbarie et de l'illégalité mais, en plus, son efficacité même est, pour la première fois, remise en question de manière explicite. Car si la torture produit le plus souvent les effets attendus, elle a, au cours de la série, connu quelques ratés. Dans la deuxième saison, Jack interroge violemment Marie Warner (Laura Harris) pour qu'elle lui révèle l'emplacement de la bombe nucléaire qu'elle prévoit de faire détonner. Pour ce faire, il lui refuse des 
antidouleurs alors que celle-ci a reçu une balle, méthode qui rappelle le scandale autour d'Abou Zubaydah ${ }^{22}$. Si Marie semble finalement craquer, elle donne de fausses informations à Jack dans le but de le tromper. Celui-ci, intuitif comme toujours, s'apercevra de la supercherie. Dans ce cas, la torture pousse à de fausses confessions, et démontre l'efficacité relative, voire l'absence d'efficacité, d'une telle méthode ${ }^{23}$. La saison 7 articule cette limite à plusieurs reprises. Ainsi, lorsque Larry découvre que Renee a torturé Tanner (Dameon Clarke), lequel a tué un suspect capital quelques heures plus tôt, il lui dit: « Tu sais aussi bien que moi que ces interrogatoires coercitifs ne sont pas fiables » (7.5).

Ce retour au droit et au respect de la loi profite aux criminels arrêtés et aux suspects récalcitrants, lesquels vont tester les limites d'un tel revirement déontologique et, par làmême, le remettre en cause. En effet, de manière récurrente et inédite, les prévenus dans la septième saison exercent leur droit à un avocat ou bien se font les défenseurs d'une loi judiciaire et morale qu'eux-mêmes ne respectent pas. Ainsi, quand Renee décide de recourir à la manière forte pour pousser Tanner à l'aveu, celui-ci s'exclame : «Vous ne pouvez pas faire ça. » Il rappelle Renee à son devoir avec un sourire narquois, conscient que la loi le protège de toute atteinte physique. Mais celle-ci, excédée par la condescendance du prisonnier, décide d'embrasser à nouveau la torture. "Vous ne pouvez pas faire ça. Vous êtes du FBI. C'est illégal. J'ai des droits », soupire Tanner avant que Renee ne le prive d'oxygène (7.4). Nous apprenons ensuite que Tanner a porté plainte contre Renee (7.5). Quelques heures plus tard, Jack torture Ryan Burnett (Eyal Podell), chef de cabinet du sénateur Mayer, dont il sait qu'il est impliqué dans l'attaque imminente contre la Maison Blanche. Alors qu'il est sur le point de révéler des informations capitales, un groupe de Marines interrompt l'interrogatoire et appréhende Jack. Livrée à Taylor, Burnett reste inflexible, refusant de coopérer, et décline même l'offre d'une grâce présidentielle :

TAYLOR. Je n'autoriserai pas la torture mais, dans votre cas, je n'aurais aucun problème avec la peine de mort.

BURNETT. Où est mon avocat?

30 Dans les deux exemples précités, les criminels se servent de la loi et du respect de celle-ci par ses représentants pour garder le silence et ne livrer aucune information. La série met en évidence la frustration et l'absurdité d'une conception purement déontologique de la loi. Dans le même temps, cette frustration est renforcée du fait de l'efficacité de la torture car Renee obtient les aveux de Tanner et Jack fait craquer Burnett. Mais, hélas, dans ce dernier cas, Jack est arrêté avant que son prisonnier n'ait pu prononcer le moindre mot. Face à un Mayer déterminé, il s'écrie : «Il allait parler! Ce qui va arriver est en cours maintenant et c'est sur votre conscience » (7.11).

31 Ainsi, même si la torture fait l'objet d'un véritable débat, la septième saison de 24 heures chrono en réaffirme l'efficacité et la nécessité en des circonstances exceptionnelles. Certes, les personnages ont conscience de son horreur et de son immoralité (mais n'est-ce pas le cas de Jack depuis le premier jour ?), pourtant, la torture continue à être perçue comme un outil de prédilection dans la lutte contre le terrorisme. Il n'est pas d'ailleurs pas étonnant que son usage se propage au fil des épisodes à de fervents opposants. Il faudra moins d'une heure à Renee pour commanditer la torture d'un suspect des mains de Jack avant de l'exécuter elle-même peu de temps après à plusieurs reprises, et moins d'une journée pour devenir un agent déchu suivant la rhétorique jusqu'au-boutiste de Jack (7.24). 
ant à Ethan, le bras droit de Taylor, il finit par épouser l'idée que la torture est nécessaire et renvoie Jack "interroger» Burnett (7.13). Et même la présidente est contrainte à reconsidérer ses positions lors d'un vif échange avec Mayer :

MAYER. Être en guerre ne justifie pas tout. (...) Vous me demandez que [Jack Bauer] ne soit plus cible de cette enquête. Et le message est clair. Dans certaines circonstances, vous trouvez ses méthodes acceptables.

TAYLOR. Il y en a qui diraient qu'elles le sont.

MAYER. Et ils auraient tort, sur les plans militaire et moral. L'Amérique est déjà

passée par là. Vous avez parlé de tragédie nationale.

La torture est donc indissociable de 24 heures chrono. Les inflexions idéologiques apportées par cette saison ont servi de réponse aux critiques sans en remettre en cause l'usage au long terme. Si elle est montrée comme nécessaire dans la guerre contre la terreur, il semble en fait qu'elle soit davantage essentielle à un niveau diégétique. Autrement dit, c'est parce que la torture est un rouage narratif capital dans l'économie de la série que cette dernière ne peut s'en passer.

En accentuant sciemment le terme "narratif ", nous réaffirmons le caractère fictionnel de la série et, ce faisant, souhaitons relativiser l'ensemble des critiques adressées aux producteurs concernant l'utilisation et l'efficacité de la torture. Faut-il préciser, comme Magritte l'a fait dans son œuvre majeure La trahison des images, que 24 heures chrono n'est en rien la réalité, que Jack Bauer n'existe pas et que ce scénario de la bombe à retardement qui dynamise sans cesse la diégèse n'est qu'un pur fantasme? Sur ce dernier point, Robert Cochran, co-créateur de 24 heures chrono, affirme que «la plupart des experts en terrorisme vous diront que ce scénario de la bombe à retardement n'arrive jamais en réalité, ou alors très rarement. Mais, dans notre série, il a lieu chaque semaine ${ }^{24}$ . » Par analogie, la torture mise en scène n'est pas de la torture mais une représentation fantasmée à des fins narratives. Car parler de torture dans 24 heures chrono, c'est avant tout parler de muthos, de mise en intrigue.

Chaque saison est bâtie en arcs autour d'une intrigue à tiroirs. De manière fractale, chaque épisode est également conçu selon cette logique. Dans cette mise en abyme vertigineuse qu'est 24 heures chrono, un élément clef permet la progression de l'intrigue : la torture. Cette dernière est donc principalement un outil narratif dont l'efficacité quasi immuable est imputable au fait que l'histoire doit nécessairement avancer à cause de sa structure fermée impliquant que tout doit être bouclé en 24 heures chrono. Ainsi, la torture apporte toujours une information décisive qui va dénouer un moment de «blocage narratif » et relancer l'intrigue. Stacy Takacs corrobore cette hypothèse, en insistant sur les liens étroits entre narration et réception: c'est parce que la série repose sur un scénario de la bombe à retardement qui, de plus, se déroule en temps réel dans une urgence permanente, que le public valide instinctivement le recours à la torture. Ce qui n'en signale pas pour autant l'adhésion morale :

La régularisation de la torture ne participe pas seulement de sa normalisation; elle pousse les spectateurs à prendre part à l'action de telle sorte qu'ils en sont presque à demander plus de torture puisque c'est la torture qui produit les informations nécessaires pour envoyer Jack sur une nouvelle piste. L'intrigue s'enraye quand la torture n'est pas utilisée, et tout débat moral sur sa légitimité ou sur son utilisation est ressenti comme un atermoiement frustrant $t^{25}$. 
Au final, si la saison 7 ne remet pas réellement en cause la torture, elle ouvre néanmoins un espace d'expression aux voix discordantes et, comme nous l'avons esquissé, oppose les deux grandes conceptions philosophiques en matière de morale que sont le déontologisme et l'utilitarisme.

\section{« Rien n'est noir ou blanc » : vers une dialectique du déontologisme et de l'utilitarisme?}

Parmi les débats animés qui ont lieu dans et autour de 24 heures chrono, celui concernant le déontologisme et l'utilitarisme est très certainement le plus important. La série pose, en effet, une myriade de dilemmes moraux qui se présentent à tous les personnages et qui leur imposent de faire le choix entre les deux théories philosophiques.

Le déontologisme est une morale conceptualisée par Emmanuel Kant dans ses Fondements de la métaphysique des moeurs de 1785 . Elle repose sur l'idée que la vertu résulte de la pureté de l'intention qui préside à une action. Pour atteindre cette intention pure et l'action morale, il faut faire preuve, selon Kant, d'une «bonne volonté», c'est-à-dire être animé par le principe même du devoir et par notre représentation de la loi morale. En tant qu'êtres conscients, nous pouvons, en effet, par le truchement de notre raison, accéder à cette loi morale innée commune à tous les hommes. Pour Kant, commettre un acte moral ne consiste donc pas à en envisager les bonnes conséquences possibles : seule l'intention compte, laquelle doit découler de notre raison. Le philosophe propose également deux impératifs catégoriques qui doivent servir de critères universels et définitifs pour la moralité de nos actions. D'une part, la règle qui nous anime doit être universalisable. D'autre part, elle ne doit jamais considérer autrui comme un moyen mais comme fin en soi.

39 Cette conception du devoir et de la morale s'oppose directement à l'utilitarisme conceptualisé par John Stuart Mill en 1863. Plus accessible que le formalisme kantien, il peut être résumé en une célèbre maxime: «la fin justifie les moyens ». Faisant partie d'une école plus vaste appelée conséquentialiste, Mill considère que «les actions sont bonnes en proportion du bonheur qu'elles donnent ${ }^{26}$. » C'est ce bonheur téléologique du plus grand nombre qui va justifier et légitimer les moyens mis en œuvre pour l'atteindre. Une telle morale reconnaît donc l'utilité du sacrifice à condition qu'il permette de servir le bien commun et de le faire prospérer.

Parce qu'il est soumis à un nombre inconsidéré de dilemmes moraux, Jack apparaît comme le cas d'étude le plus fascinant de la série. Celui-ci a d'ailleurs fait l'objet de nombreuses analyses tendant à le faire apparaitre comme l'archétype de l'utilitariste. Ainsi, pour Thibaut de Saint Maurice, Jack est un anti-kantien car il « n'est pas un homme de devoir ${ }^{27}$ » au sens où il ne respecte pas les impératifs moraux de l'éthique déontologique, notamment en matière de respect de l'humanité. Il affirme que le héros de 24 heures chrono "se soucie bien peu de la forme de son action et de la pureté de son action. Seul le résultat compte, toujours et encore, le résultat ${ }^{28}$. »

Ce constat implacable renvoie aux innombrables cas où Jack choisit de sacrifier une personne, un groupe de personnes, au nom du bien commun. Dans la saison deux, il n'hésite pas à mettre la vie de Kate Warner (Sarah Wynter) et de Sherry Palmer (Penny Johnson Jerald) en danger afin, respectivement, de retrouver un terroriste présumé et d'empêcher la destitution définitive de David Palmer (Dennis Haysbert). Dans la saison 
trois, il exécute de sang-froid Ryan Chappelle (Paul Schulze), l'un de ses supérieurs à la CTU, pour satisfaire aux exigences du terroriste Stephen Saunders (Paul Blackthorne). Plus tard, dans la saison six (6.15), il risque la vie de Brady Hauser (Scott Michael Campbell), le frère autiste d'un suspect, afin de retrouver Gredenko (Rade Šerbedžija), un nationaliste russe malfaisant. S'il est prêt à sacrifier autrui pour le salut de l'Amérique, il est aussi prêt à se sacrifier. Il accepte, par exemple, de troquer sa vie contre les informations que détient Nina Myers (Sarah Clarke) sur la bombe nucléaire qui menace d'exploser (2.10) et d'être livré à Fayed dans un contexte similaire (6.1). À cette occasion, Jack révèle à Bill (James Morrison) que : «aujourd'hui, je peux mourir pour quelque chose. De la manière dont je veux et selon mon consentement. » Jack affirme qu'il a fait le choix de ne pas mourir dans les mains de ses geôliers chinois parce que cela aurait été un sacrifice vain. Se sacrifier, oui, mais pour le bien commun, maxime en parfaite adéquation avec la doctrine utilitariste ${ }^{29}$. Pour autant, est-il uniquement un « utilitariste obstiné ${ }^{30} »$ ? Jean-Baptiste Jeangène-Vilmer donne une première réponse péremptoire: "les (mauvaises) analyses philosophiques de 24 présentent souvent Jack comme l'archétype de l'utilitariste ${ }^{31}$.» Pour lui, la notion d'archétype en matière d'utilitarisme et de déontologisme ne s'applique pas aux personnages de la série, ou alors uniquement aux personnages secondaires, car, du fait du format dans lequel ils évoluent, ils se doivent d'être complexes et instables. Au regard des neuf saisons de 24 heures chrono, il est légitime de dire que la série évite l'écueil de la caricature et du manichéisme. JeanBaptiste Jeangène-Vilmer montre, au fil d'une analyse très convaincante, qu'il existe en fait des utilitaristes et des déontologistes «de seuil » qui vont, en des circonstances particulières, lorgner vers la théorie morale opposée. Une telle conception implique que les modèles de pensée ne valent qu'à un niveau abstrait. Dès lors qu'on les objectivise, qu'ils se trouvent confrontés à notre monde sensible, la division théorique ne tient plus car la complexité du monde rend intenable une posture définitive.

Suivant ce constat, Jack ne peut pas seulement être utilitariste. Jean-Baptiste Jeangène Vilmer remarque qu'il est, en effet, parfois le défenseur de la morale déontologique pour trois raisons principales ${ }^{32}$. D'abord, Jack n'est pas impartial (ce qu'exige le calcul utilitariste) puisqu'il lui arrive de sauver des vies sans penser aux conséquences. Ensuite, Jack est un homme de parole. Le plus souvent, lorsqu'il fait une promesse, il s'y tient, ce qui est fondamentalement kantien. Enfin, Jack croit en la justice rétributive, autrement dit que justice soit faite coûte que coûte a posteriori, ce qui s'oppose à une vision préventive, donc utilitariste, de la justice.

Brett Chandler Patterson soutient également l'idée d'une complexité plus grande du personnage en arguant qu' 'à bien des égards Jack apparaît comme le prototype de l'utilitariste ; il cherche toujours à atteindre le souverain bien pour le plus de personnes possibles. Néanmoins, cette simple caractérisation est trop restrictive. ${ }^{33}$ » Il ajoute une distinction pertinente qui démontre que Jack est moralement plus nuancé puisqu'il souligne que celui-ci est uniquement utilitariste lorsqu'il s'agit de sauver l'Amérique.

En revanche, dès lors qu'il s'agit de sa famille, Jack est immuablement déontologique au sens où la défense de la famille apparaît comme un impératif catégorique kantien. L'exemple le plus flagrant se déroule dans l'épilogue de la saison six. Le vice-président Noah Daniels (Powers Boothe) vient d'accepter de livrer Josh (Evan Ellingson), neveu de Jack, à son grand-père malfaisant Phillip (James Cromwell) en échange d'un module à l'importance capitale pour le maintien de la paix américano-russe. Josh se retrouve donc sacrifié par la nation américaine au nom du bien commun, un calcul utilitariste qui 
révolte Jack et qui le pousse à agir à l'encontre de l'ordre présidentiel Patterson note que, dans cette situation précise :

Jack agit davantage selon une éthique du devoir (déontologisme) et s'oppose aux utilitaristes sans cœur de la Maison-Blanche. (...) Quand il se tourne vers les siens, il se rend compte que son désir d'intimité et son sens de la loyauté l'empêchent d'être un utilitariste forcené ${ }^{34}$.

46 comme « une perte acceptable » (6.24), mais son lien de parenté maintient le doute sur ses motivations. Que n'a-t-il pas commis, en effet, pour sauver sa fille Kim (Elisha Cuthbert) ou sa petite amie Audrey (Kim Raver)? En fait, une interprétation déontologique du rapport de Jack à sa famille semble fallacieuse puisqu'elle omet les motifs affectifs qui motivent son action. Autrement dit, lorsqu'il met tout en œuvre pour sauver les siens, Jack n'est pas seulement motivé par son devoir et le respect d'impératifs moraux (il ne faut pas laisser mourir un innocent) puisqu'il est également animé par l'amour qu'il porte à sa famille. Cette posture ambiguë est en désaccord avec l'éthique kantienne.

On peut dès lors affirmer que Jack agit au sein d'une pluralité de valeurs et de modèles moraux. Si ses actions semblent osciller entre utilitarisme et respect de grands principes universels, Jack applique une morale opératoire qui le pousse à s'adapter : grâce à son expérience du réel et aux informations dont il dispose, il parvient à trouver ce qui lui paraît être la meilleure solution - la plus efficace - face à l'urgence d'une situation impossible. Jack n'a donc souvent ni le les conséquences d'un point de vue moral : pris dans un déferlement de dilemmes inextricables, il doit tout bonnement agir selon son instinct et ses sentiments. Il rappelle cette nécessité de s'adapter et de répondre rapidement à une situation donnée lors de son audition dans l'épisode 7.1 :

JACK : La vérité, sénateur, c'est que j'ai empêché cet attentat.

MAYER : En torturant Monsieur Haddad?

JACK : En faisant ce qui m'a paru nécessaire pour sauver des innocents. (...) Pour un soldat, la différence entre la réussite et l'échec tient en sa capacité à s'adapter à l'ennemi. Ceux que je combats n'ont que faire de vos règles. Tout ce qui leur importe, c'est le résultat. Mon travail, c'est de les empêcher d'accomplir leurs objectifs. Je me suis simplement adapté. (je souligne)

Ces remarques nous amènent à envisager une autre morale permettant de mieux comprendre le comportement de Jack: le pragmatisme. Ce concept philosophique dont on attribue la parenté aux penseurs américains de la fin du XIXe siècle Charles S. Peirce et William James définit une morale qui ne vise pas tant le respect d'une règle métaphysique a priori ou une finalité particulière que la recherche par l'individu des meilleures solutions face à l'imprévisible ${ }^{35}$. La moralité de ces dernières peut être jugée par la suite à l'aune de leurs résultats et de leurs effets. Jack en est d'ailleurs conscient et il est prêt à être jugé pour ses actes par ceux-là même qu'il n'a cessé de sauver. Il l'affirme au cours de la scène citée plus tôt : «Suis-je au-dessus des lois? Non, monsieur. Et je suis tout à fait disposé à être jugé par ces citoyens que vous prétendez représenter. »

L'apport de la philosophie pragmatique nous permet alors de préciser le constat de Brett Chandler Patterson pour qui Jack incarne un « martyr utilitariste ${ }^{36}$ ». En se lançant « dans des actions qui sont 'nécessaires' selon sa vision du monde ${ }^{37}$ », il est davantage un «martyr pragmatique». Cette posture qu'il adopte est un fardeau qui le contraint à se salir les mains à la place des autres. «Les gens comme vous veulent des résultats mais 
vous ne voulez jamais avoir les mains sales ", lance-t-il à George dans la saison deux (2.1). Jack embrasse le mal, non pas parce qu'il est mauvais, mais parce que cela est nécessaire: il fait ce qui doit être fait en tant que soldat et en temps de guerre.

La septième saison de la série met en scène de façon consciente le débat déontologisme versus utilitarisme. Comme nous l'avons vu plus haut, les temps ont changé, l'Amérique est à nouveau régie par la loi et le respect de la loi, qu'elle soit judiciaire ou morale. Sous la présidence d'Allison Taylor, la nation retrouve des aspirations déontologiques. Cela se remarque par l'arrivée d'un nombre important de personnages kantiens, Larry Moss, Renee Walker, Janis Gold et, bien sûr, le sénateur Mayer. Avec la présidente Taylor, ils représentent ce fantasme de l'intention pure et ils sont mus par leur sens aigu du devoir. Leur confrontation avec Jack, qui en ce sens ne serait que l'incarnation du principe de réalité, provoque néanmoins des migrations sur l'axe déontologisme/utilitarisme alors que la menace terroriste se fait plus virulente et que les dilemmes moraux se multiplient. Ainsi, nous avons choisi de récapituler ces inflexions idéologiques à travers la figure cidessous :

Fig. 1 : représentation des inflexions au cours de la saison 7 à partir du placement idéologique originel (7.1) des personnages

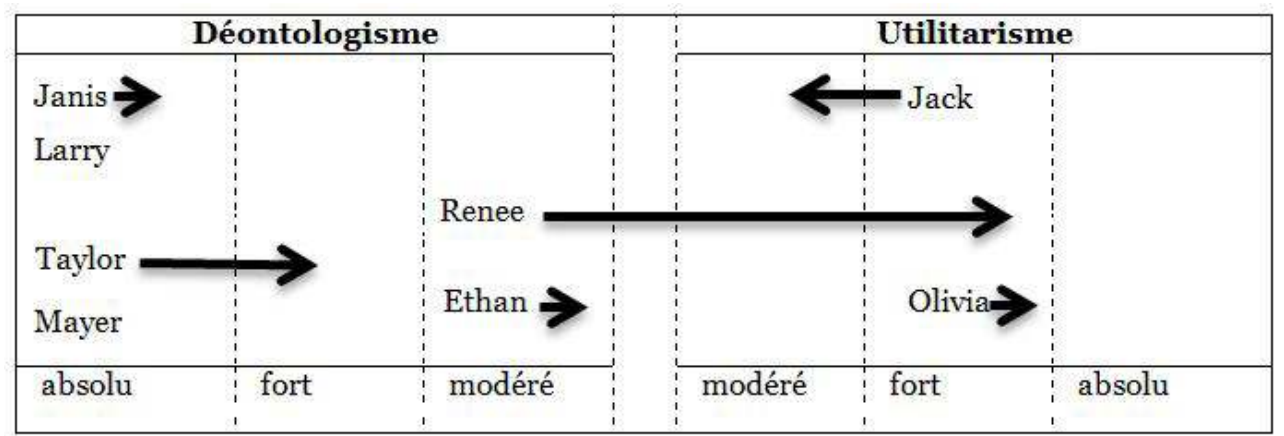

Figure 1: représentation des inflexions au cours de la saison sept à partir du placement idéologique originel (7.01) des personnages.

51 Mis à part Larry Moss et Mayer, qui apparaissent comme des figures absolues du déontologisme, tous les autres personnages à obédience kantienne sont attirés vers la morale utilitariste à des degrés différents. Cela semble traduire ce que nous évoquions plus tôt sur la rupture entre théorie et pratique, entre principes universels et expérience du réel. La morale de Kant, en tant que concept, fonctionnerait si tout un ensemble de paramètres étaient rassemblés. Vivre de manière déontologique suggère non seulement que je respecte la morale mais aussi qu'autrui la respecte. Sinon, le principe même d'universalité des actions ne tient plus.

La morale déontologique ne peut se produire que dans un monde utopique où les rapports entre les individus sont déjà moraux, au sens où chacun choisit de respecter la loi morale et autrui. Ce monde utopique, c'est en quelque sorte le hors champ inaugural de la saison 7 de 24 heures chrono. Les conditions sont réunies pour entretenir une morale déontologique. Mais que se passe-t-il dès lors que, sur le terrain, ce schéma de respect mutuel s'érode et que certains individus choisissent de ne plus respecter la loi ? Comment agir de manière déontologique dans un contexte réel de non-réciprocité ? C'est justement à cette question que répond la série en montrant comment la morale de Kant se heurte à 
la complexité d'un monde où « tout n'est pas blanc ou noir », comme le confie un imam à Jack (7.24).

53 Le parcours de la présidente Taylor semble à cet égard particulièrement intéressant. Elle se présente comme une déontologue qui a remis l'Amérique sur le chemin de l'exigence morale. Quand, à la fin de la saison, elle doit faire face à un cas de conscience déchirant et décider si oui ou non elle fera incarcérer sa fille, laquelle a commandité l'assassinat de Jonas Hodges (Jon Voight), la présidente fait le choix de la justice et livre Olivia (Sprague Grayden) aux autorités quitte à détruire sa famille (on apprend dans la saison huit qu'elle a divorcé de son mari). Malgré tout, au fil des heures, la morale conséquentialiste incarnée par Jack semble l'atteindre, notamment lorsqu'elle fait prévaloir le principe de non-négociation avec les terroristes qui tient de l'utilitarisme de la règle (il ne faut pas négocier avec des terroristes sinon ils recommenceront et cela fera beaucoup plus de victimes). Ainsi, lorsqu'elle refuse de replier sa flotte aérienne loin du Sangala, le général Juma (Tony Todd) met à exécution sa menace et fait entrer en collision deux avions civils avec à bord plusieurs centaines d'innocents (7.6).

Par ailleurs, son discours en matière de torture évolue également au fil de la saison. D'abord foncièrement opposée, elle va néanmoins tenter de convaincre Mayer de gracier Jack avouant qu' " au cours des dix dernières heures, les choses ne sont pas apparues aussi noire et blanche qu'elles ont pu l'être » (7.11). Et quand Jack est arrêté après avoir torturé Burnett, elle se surprend à reconsidérer l'acte en lui-même: «Bauer qui va en prison alors qu'un traitre s'en tire. Qu'est-ce qui ne va pas dans ce tableau? »

Jack, quant à lui, semble emprunter un cheminement inverse. Il commet ainsi des entorses à la morale utilitariste dans un moment clef de la saison lorsqu'il sauve un agent de sécurité d'une mort certaine quitte à ne pas remettre la main sur une arme bactériologique. Tony lui lance alors : « Ne viole pas tes propres règles. Tu vas peut-être sauver un homme mais qu'en est-il de tous ceux qui pourraient mourir dans une attaque biologique? » (7.15) Plus tard, lorsque Kim est (encore) kidnappée par des terroristes, il cède aux exigences de ses derniers et accepte de libérer Tony (7.23). Bien sûr, il a toujours recours à la torture et utilise encore des innocents pour parvenir à ses fins mais Jack est prêt à faire le choix du Bien et à privilégier les principes au détriment des résultats. Son dernier échange avec Mayer est en cela éloquent puisque les deux hommes arrivent enfin à se comprendre et à trouver un terrain d'entente. Jack lui fait part de ses remords, lui confiant qu'il regrette plus que tout que «ce monde ait même besoin de gens comme moi » (7.13). Mayer a conscience de tout ce qu'il a sacrifié au nom du bien commun et lui assure qu'à présent il doit faire confiance aux institutions américaines et emprunter le chemin de la morale kantienne :

MAYER. Il est temps que vous commenciez à faire confiance aux institutions pour lesquelles vous avez tant sacrifié afin de les protéger.

JACK. La confiance n'est pas mon point fort.

MAYER. Mon garçon, il faut commencer quelque part.

Ironie tragique pour Jack, celui qui lui promet la chance d'un renouveau sera tué sous ses yeux quelques secondes plus tard, le contraignant à revenir à ses méthodes traditionnelles. 24 heures chrono semble donc bien réaffirmer qu'en des circonstances exceptionnelles (i.e. état d'exception), respecter des principes moraux universels et intangibles est intenable car cela ne permet pas d'affronter, par exemple, la complexité du terrorisme actuel. Cela se confirme à nouveau dans l'épilogue de la saison (7.24). Renee vient d'arrêter Alan Wilson (Will Patton) et le suspecte de cacher des informations sur 
une possible nouvelle attaque. Elle vient demander conseil à Jack sur ce qu'elle doit faire, soit utiliser la torture pour lui soutirer ce qu'il sait, soit le laisser aux mains de la justice quitte à mettre l'Amérique en danger. Jack lui répond avec une parabole :

JACK. Je vois quinze otages dans un bus et j'oublie tout le reste. Je ferai tout pour les sauver. Absolument tout. Peut-être que je me dis qu'en les sauvant, c'est moi que je sauve. (...) [Les] lois ont été établies par des hommes plus intelligents que moi. Et en définitive, je sais qu'elles sont plus importantes que les quinze personnes dans le bus. Dans mon esprit, je sais que c'est très bien ainsi. Mais mon cœur ne peut le supporter.

57 À travers cet exemple, Jack révèle qu'il est profondément convaincu que les règles déontologiques devraient être respectées, mais que la réalité de la guerre contre la terreur et ses propres sentiments le forcent sans cesse à transiger. Même si sauver des vies est une maxime universalisable, recourir à tous les moyens possibles, dont la torture, revient à se placer en-dessous de l'humanité, ce qui va à l'encontre de la doctrine kantienne. Jack en a bien conscience : il sait qu'il est probablement plus moral de laisser mourir des innocents que de piétiner les lois de la Cité. Il procède ainsi d'une dichotomie. La déontologie appartiendrait au domaine de l'esprit, de la raison, tandis que l'utilitarisme serait davantage le fruit des émotions, du cœur. Parce que les journées infernales de 24 heures chrono déroulent un flux diluvien de dilemmes moraux qui ne laissent pas le temps de la contemplation intellectuelle, elles appellent nécessairement une réponse émotive. Cela justifie la décision de Jack d'épouser sa logique du « whatever it takes » dans le cas du bus et des quinze otages qui sont à bord : il ne peut vivre avec leur mort.

La saison 7 insiste donc sur le fait que Jack n'est pas essentiellement utilitariste. Il apparaît davantage comme un adepte du pragmatisme en quête perpétuelle d'une action adaptative et utilitaire permettant de répondre efficacement au terrorisme. Il s'efforce ainsi de respecter des principes moraux, mais il est contraint de s'adapter face à la réalité de la guerre, à l'état d'exception et aux multiples dilemmes qu'il rencontre en allant trouver, dans l'urgence et avec les informations à sa disposition, les solutions qui lui semblent humainement les meilleures.

\section{Conclusion : continuités diégétiques, continuités historiques}

Si la septième saison se place d'emblée sous le signe du renouveau pour des raisons artistiques et économiques, ce sont surtout ses inflexions idéologiques marquantes qui en font une pierre angulaire dans l'histoire de 24 heures chrono. Osant désormais ausculter de manière claire et directe ses tensions internes, la série remet en cause son biais utilitariste et se confronte à la critique. En incorporant des personnages plus moraux, au sens kantien, elle fait de Jack un criminel, un monstre anachronique qui n'a plus de place dans une Amérique à nouveau régie par le droit et la morale. Jack semble incarner alors une autre Amérique, celle des années Bush, dont l'héritage est renié lors de l'élection de Barack Obama. La rhétorique utilitariste n'a plus lieu d'être, la torture est désormais vue comme immorale et illégale. Pour preuve, l'annonce de la fermeture de Guantanamo par le président démocrate lors de sa campagne. L'Amérique a changé, 24 heures chrono aussi.

Pourtant, ce postulat n'est pas satisfaisant car si la série opérait un véritable changement, la morale kantienne remplacerait finalement la morale utilitariste. Or, celle-ci est 
réaffirmée et les déontologues semblent progressivement s'en rapprocher ou, s'ils campent sur leurs positions, y laissent la vie (ex : Mayer, Larry). Le leitmotiv de la série reste en cela univoque : l'utilitarisme est la seule morale efficace à adopter pour contrer la menace terroriste et permettre le sauvetage du plus grand nombre. De fait, sa remise en cause dans la série est un leurre. La torture, bien que faisant l'objet de critiques, reste ainsi un moyen fiable afin d'obtenir des informations capitales même si son exercice n'est pas sans conséquences.

61 La septième saison se placerait donc dans la continuité des précédentes, aussi bien formellement qu'idéologiquement. Malgré les fortes dissonances que l'on peut y entendre et qui peuvent pousser le spectateur à revoir son rapport aux actes de Jack, 24 heures chrono réitère son discours. Ainsi, la série s'adapte-t-elle vraiment à l'ère Obama? Pour répondre à une telle question, il faut s'attarder un instant sur les deux mandats du président américain en exercice depuis 2009. Force est de constater que les changements radicaux promis par Obama lors de sa campagne n'ont pas eu lieu. Comme le souligne Thomas Rabino, «le bouleversement à la Maison-Blanche relevait davantage du rêve, voire de l'idéologie, que du principe de réalitée ${ }^{38}$. "Si Obama a bien mis un terme à l'enlisement en Irak, il a néanmoins augmenté la présence militaire américaine en Afghanistan. Guantanamo, dont il avait promis la fermeture, est aujourd'hui toujours ouvert. Le PATRIOT Act, loin d'avoir été abandonné, a été reconduit bien que certaines inflexions lui aient été apportées en 2015. Nombreux sont aujourd'hui les exemples symboliques d'une continuité avec la politique bushiste. Ainsi, la saison 7 et les suivantes semblent bien représenter l'indifférenciation politique en matière de réponse au terrorisme et, finalement, l'idéologie en partage des camps démocrate et républicain aux États-Unis.

\section{BIBLIOGRAPHIE}

BENTHAM, Jeremy, "Of Torture", manuscrit transcrit par W.L. et P.E. Twining dans « Bentham on Torture », in B. Parekh (éd.) Jeremy Bentham: Critical Assessments, vol. II, Londres, Routledge, 1993, 599 p.

CALDWELL, Anne et Samuel Chambers « 24 after 9/11: The American State of Exception », in Steven Peacock (ed.), Reading 24, TV against the clock, New York, I.B. Tauris, 2007, p. 97-108.

DE SAINT-MAURICE, Thibault, Philosophie en séries, Paris, Ellipses, 2009.

DUNN, Timothy, « Torture, Terrorism and 24: What Would Jack Do? », in J. J. Foy (ed.) Homer Simpson Goes to Washington: American Politics through Popular Culture, Lexington, University of Kentucky Press, 2008, p. 171-184.

Edelstein, David, « Now Playing at Your Local Multiplex: Torture Porn », New York Magazine, 6 février 2006.

FALUDI, Susan, The Terror Dream. What 9/11 Revealed About America, Londres, Atlantic Books, 2008. 
FENTON, Jenifer, « Former Guantanamo inmates tell of confessions under 'torture'« , cnn.com, 28 octobre 2011. Disponible à : http://edition.cnn.com/2011/10/28/world/meast/guantanamoformer-detainees/

GREENE, Eric, « Jack Bauer Syndrome », in Richard Miniter (ed.) Jack Bauer for President, Terrorism and Politics in 24, Dallas, Benbella, 2008, p. 171-193.

HOWARD, Douglas L., "'You're going to tell me everything you know', Torture and Morality in Fox's 24 », in Steven Peacock (ed.) Reading 24, TV against the clock, New York, I.B. Tauris, 2007, p. $133-145$.

JAMES, William, Pragmatism, Mineola, Dover, 1995.

JEANGÈNE VILMER, Jean-Baptiste, 24 heures chrono. Le choix du mal, Paris, PUF, 2012.

LEVINSON, Jerrold, «Contextualisme esthétique », in Martin Montminy (ed.) Philosophiques, vol. 32, $\mathrm{n}^{\circ} 1,2005$, p. 125-133.

MILL, John Stuart, L'utilitarisme, Paris : Flammarion, 1988 (première publication en 1863), 181 p.

NATTA, Don Van, "Questioning Terror Suspects in a Dark Surreal World », The New York Times, 9 mars 2003. Disponible à : http://www.nytimes.com/2003/03/09/international/09DETA.html/? _r $=0$

PATTERSON, Brett Chandler, «I Despise You for Making Me Do This ", in Richard Miniter (ed.) Jack Bauer for President, Terrorism and Politics in 24, Dallas, Benbella, 2008, p. 29-41.

PICHARD, Alexis, Le nouvel âge d'or des séries américaines, Paris, Le Manuscrit, 2013 (2 $2^{\text {nde }}$ éd.).

PICHARD, Alexis, « Patty, Vic, Jack et les autres : antihéros modernes et postmodernes dans les séries américaines contemporaines », in Ariane Hudelet et Sophie Vasset (ed.) TV/Series, Les Séries télévisées américaines contemporaines : Entre la fiction, les faits, et le réel, $\mathrm{n}^{\circ} 1$, juin 2012, p. 513-530.

RABINO, Thomas, De la guerre en Amérique, Paris, Perrin, 2011.

SCAHILL, Jeremy, Dirty Wars: the World is a Battlefield, Londres, Serpent trail, 2013.

STELTER, Brian, « Creator of '24' Calls It Quits », The New York Times, 14 février 2008. Disponible à : http://mediadecoder.blogs.nytimes.com//2008/02/14/creator-of-24-calls-it-quits/ SUTHERLAND, Sharon et Sarah Swan, «'Tell me where the bomb is or I will kill your son', Situational Morality on 24 », in Steven Peacock (éd.), Reading 24, TV against the clock, New York, I.B.Tauris, 2007, p. 119-132.

TAKACS, Stacy, Terrorism TV, Popular Entertainment in Post-9/11 America, Lawrence, University Press of Kansas, 2012.

\section{NOTES}

1. Jerrold Levinson, «Contextualisme esthétique », in Martin Montminy (dir.) Philosophiques, vol.32, $\mathrm{n}^{\circ} 1,2005, \mathrm{p} .128$.

2. Documentaire disponible sur le coffret DVD de la saison sept paru chez Fox entertainment (2009).

3. Diffusé le 14 janvier 2007.

4. Diffusé le 21 mai 2007.

5. «24/7 : the Untold Story », op. cit. (toutes les traductions, sauf mention contraire, sont de l'auteur) 
6. Jean-Baptiste Jeangène Vilmer, 24 heures chrono : le choix du mal, Paris : PUF, 2012, p.131.

7. Jane Mayer, "Whatever it takes: The Politics of the man behind 24 », New Yorker, 19 février 2007.

8. Ibid.

9. Jeangène Vilmer, op. cit., p.133-134.

10. Brian Stelter, "Creator of '24' Calls It Quits », The New York Times, 14 février 2008. Disponible à: http://mediadecoder.blogs.nytimes.com//2008/02/14/creator-of-24-calls-it-quits/ (consulté le 2 février 2016)

11. Jeremy Scahill, Dirty Wars : the World is a Battlefield, London, Serpent trail, 2013, p.244-247.

12. Discours disponible à: https://www.whitehouse.gov/blog/2009/01/21/president-barackobamas-inaugural-address (consulté le 16 mars 2016)

13. Alexis Pichard, Le nouvel âge d'or des séries américaines, Paris, Le Manuscrit, 2013 (2 ${ }^{\text {nde }}$ éd.), p.73-79.

14. Stacy Takacs, Terrorism TV, Popular Entertainment in Post-9/11 America, Lawrence, University Press of Kansas, 2012, p.144-145.

15. Susan Faludi, The Terror Dream, What 9/11 Revealed About America, Londres, Atlantic Books, 2008, p.139.

16. Stacy Takacs, op. cit., p.85.

17. David Edelstein, « Now Playing at Your Local Multiplex : Torture Porn », New York Magazine, 6 février 2006.

18. Eli Lake, "The Reality of Interrogation ", in Richard Miniter (éd.) Jack Bauer for President, Terrorism and Politics in 24, Dallas, Benbella, 2008, p.125-131.

19. Bentham, Jeremy, "Of Torture", manuscrit transcrit par W.L. et P.E. Twining dans « Bentham on Torture ", in B. Parekh (éd.) Jeremy Bentham: Critical Assessments, vol. II, Londres, Routledge, 1993, p.514.

20. Timothy Dunn, «Torture, Terrorism and 24 : What Would Jack Do ? », in J. J. Foy (éd.) Homer Simpson Goes to Washington: American Politics through Popular Culture, Lexington, University of Kentucky Press, 2008, p.175.

21. Pour l'anecdote, le sénateur a été nommé d'après la journaliste Jane Mayer, indiquant clairement l'intention des auteurs de la série : répondre aux critiques.

22. Abou Zubaydah, lieutenant supposé d'Al-Qaïda, fut capturé par l'armée américaine au Pakistan en 2002. Blessé par balle à l'aine lors du raid ayant mené à son arrestation, il ne reçut les soins nécessaires, particulièrement les antidouleurs, qu'après avoir accepté de coopérer lors des phases d'interrogatoire. (Don Van Natta, "Questioning Terror Suspects in a Dark Surreal World », The New York Times, 9 mars 2003. Disponible à: http://www.nytimes.com/2003/03/09/ international/09DETA.html/?_r=0 (consulté le 16 février 2016))

23. Jenifer Fenton, « Former Guantanamo inmates tell of confessions under 'torture' », cnn.com, 28 octobre 2011. Disponible à: http://edition.cnn.com/2011/10/28/world/meast/guantanamoformer-detainees/ (consulté le 16 février 2016)

24. Jane Mayer, op. cit.

25. Stacy Takacs, op. cit., p.93.

26. John Stuart Mill, L'utilitarisme, Paris, Flammarion, 1988 (première publication en 1863).

27. Thibaut de Saint Maurice, op. cit., p.19.

28. Ibid.

29. L'intention louable et désintéressée du sacrifice chez Jack peut néanmoins être remise en cause : «en réalité, cette posture quasi-christique ne sert qu'à cacher le fait que, derrière des intentions en apparence altruistes, Jack Bauer n'est pas dénué de considérations égoïstes comme il le révèle dans Rédemption, le téléfilm servant de prologue à la 7e journée («Maybe I thought if I saved them, I'd save myself.»). En définitive, si Bauer utilise tous les moyens pour servir l'intérêt général, c'est aussi parce qu'il recherche son intérêt particulier. On peut d'ailleurs douter de la 
réalité de ses propres sacrifices. Car, après le meurtre originel de son épouse Terri, notre personnage a-t-il vraiment encore quelque chose à perdre ? Les sept saisons suivantes n'auront de cesse de nous le montrer sous l'angle d'un être dépressif qui, après un passage par la drogue, élabore des plans beaucoup plus suicidaires que véritablement altruistes. " (Alexis Pichard, «Patty, Vic, Jack et les autres : antihéros modernes et postmodernes dans les séries américaines contemporaines", in Ariane Hudelet et Sophie Vasset (éd.) TV/Series \#1, Les Séries télévisées américaines contemporaines : Entre la fiction, les faits, et le réel, juin 2012.)

30. Thibaut de Saint Maurice, op. cit., p.20.

31. Jean-Baptiste Jeangène Vilmer, op. cit., p.82.

32. Ibid, p.84-88.

33. Brett Chandler Patterson, «I Despise You for Making Me Do This », in Richard Miniter (éd.) Jack Bauer for President, Terrorism and Politics in 24, Dallas, Benbella, 2008, p.35.

34. Ibid.

35. William James, Pragmatism, Mineola, Dover, 1995.

36. Ibid, p.37.

37. Ibid.

38. Thomas Rabino, De la guerre en Amérique, Paris : Perrin, 2011, p.435.

\section{RÉSUMÉS}

Cet article se propose d'analyser l'évolution en termes moraux et éthiques de la série 24 heures chrono (Fox, 2001-2010 ; 2014) à l'aune de sa septième saison. Jusque-là accusée d'épouser la politique antiterroriste utilitariste menée par l'administration du président Républicain en exercice George W. Bush, la série amorce un tournant idéologique à l'occasion de sa septième saison, dont la diffusion débute quelques jours avant l'investiture du Démocrate Barack Obama en janvier 2009. Les grandes polémiques qui entourent 24 heures chrono - en premier lieu, la surreprésentation de la torture - font désormais l'objet de profonds questionnements au sein même de la diégèse et alimentent un débat philosophique plus global quant à l'éthique à adopter dans la guerre contre le terrorisme. Sont alors opposés le déontologisme kantien et de l'utilitarisme théorisé par Jeremy Bentham. Cet article s'attache ainsi à mettre au jour les tensions idéologiques qui animent la septième saison de 24 heures chrono et à évaluer la mesure des inflexions que celle-ci opère dans le sillage de l'élection d'Obama.

This article seeks to analyse the moral and ethical evolutions in TV series 24's seventh season. In its first years, the show was accused of overtly supporting the utilitarian antiterrorism policy of the Bush administration. Still, it arguably initiated an ideological reorientation in its seventh season which started airing in January 2009 just a few days before Obama's inaugural address. All the controversies surrounding the show - the first of which being its highly criticised depiction of torture - were now being thoroughly questioned within the diegesis, which fuelled an overall philosophical debate on which ethics to espouse in the War on Terror. Two normative ethical theories were opposed, namely Emmanuel Kant's deontology and Jeremy Bentham's utilitarianism. Hence, this article highlights the ideological tensions at work in 24's seventh season and assesses the extent to which the show actually departed from its original ethical stand in the wake of Obama's election. 
INDEX

Keywords : 24, War on Terror, ethics

Mots-clés : 24 heures chrono, guerre contre la terreur, morale, Bush George W., Obama Barack, torture

\section{AUTEUR}

\section{ALEXIS PICHARD}

Agrégé d'anglais et ATER à l'université Paris Ouest. Alexis Pichard prépare une thèse à l'université du Havre sur l'ambiguité des représentations de la guerre contre la terreur dans les thrillers politiques 24 heures chrono et Homeland. Il a déjà consacré plusieurs ouvrages aux séries télévisées américaines, parmi lesquels Les séries américaines, la société réinventée ? (L'Harmattan, 2013), codirigé avec Aurélie Blot. 